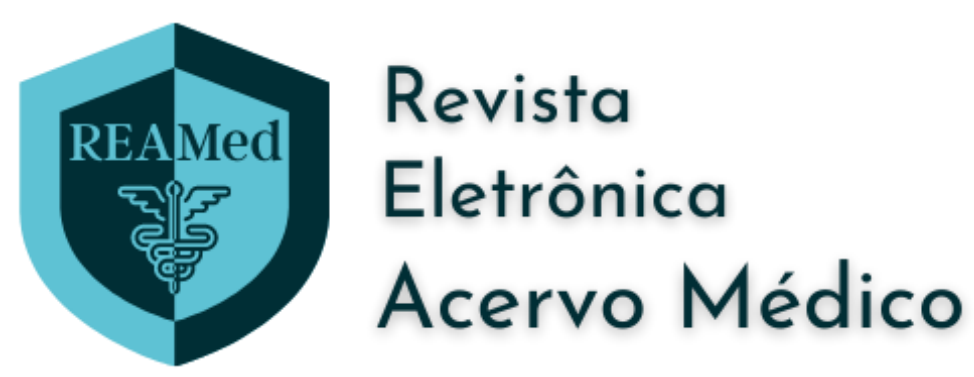

\title{
Epidemiologia e tratamento da Esofagite Eosinofílica: uma revisão narrativa
}

\author{
Epidemiology and treatment of Eosinophilic Esophagitis: a narrative review
}

Epidemiología y tratamiento de la Esofagitis Eosinofílica: una revisión narrativa

Pedro Henrique Pinto Martins ${ }^{1 *}$, Anna Luiza Guimarães Fraga1, Fernanda Souto Carvalho ${ }^{1}$, Gustavo Martins de Araujo Porto ${ }^{1}$, Isadora Nunes Delaia ${ }^{1}$, Mikaela de Miranda Lopes ${ }^{1}$, Renzo Curty Breves ${ }^{1}$, Thales Montela Marins ${ }^{1}$, Yago Souza Luiz¹, Renan Alexandre Baptista Bonancim¹.

\section{RESUMO}

Objetivo: Revisar de forma didática a abordagem geral da Esofagite Eosinofílica (EEo), discutindo desde a epidemiologia até o tratamento. Revisão bibliográfica: A doença é caracterizada pelo acúmulo de eosinófilos no esôfago e possui maior incidência nos adultos. A fisiopatologia da EEo está ligada a fatores genéticos, ambientais e alérgenos, que atraem eosinófilos e linfócitos $T$ para a região. As células $T$ helper 2 são estimuladas a produzirem interleucinas, as quais ativam eosinófilos e estimulam produção de eotaxina-3 e redução da produção de filagrina e desmogleína 1 , enfraquecendo a barreira epitelial e facilitando a inflamação. Crianças possuem dificuldade de crescimento e ganho de peso, enquanto adultos possuem disfagia, regurgitação e plenitude. O diagnóstico se dá pela presença de 15 eosinófilos em pelo menos uma das seis amostras de biópsias esofagianas realizadas por via endoscópica. A primeira linha de tratamento é usada com Inibidores da Bomba de Prótons e corticosteroides, junto com dietas. A dilatação mecânica por endoscopia e o uso de biológicos faz-se necessário quando o paciente não responde a terapia. Considerações finais: A EEo ainda é uma enfermidade de difícil diagnóstico com presença de sintomas confundidos com Doença do Refluxo Gastroesofágico. Quando presente deve ser rapidamente tratada, evitando complicações.

Palavras-chave: Esofagite eosinofílica, Gastroenterologia, Diagnóstico.

\begin{abstract}
Objective: Didactically review the general approach to Eosinophilic Esophagitis (EEo), discussing from epidemiology to treatment. Bibliographic review: The disease is characterized by the accumulation of eosinophils in the esophagus and has a higher incidence in adults. The pathophysiology of E Eo is linked to genetic, environmental and allergenic factors, which attract eosinophils and T lymphocytes to the region. $T$ helper 2 cells are stimulated to produce interleukins, which activate eosinophils and stimulate production of eotaxin-3 and reduced production of filaggrin and desmoglein 1, weakening the epithelial barrier and facilitating inflammation. Children have growth difficulties and weight gain, while adults have dysphagia, regurgitation and fullness. The diagnosis is made by the presence of 15 eosinophils in at least one of the six samples of esophageal biopsies performed endoscopically. The first line of treatment is used with Proton Pump Inhibitors and corticosteroids, along with diets. Mechanical dilation by endoscopy and the use of biologics are necessary when the patient does not respond to therapy. Final Considerations: EEo is a disease of difficult diagnosis with the presence of symptoms associated with Gastroesophageal Reflux Disease. When present, it must be quickly treated as a complication.
\end{abstract}

Key words: Eosinophilic esophagitis, Gastroenterology, Diagnosis.

1 Universidade de Vassouras (UV), Vassouras - RJ. *E-mail: pedrohmpbi@gmail.com

SUBMETIDO EM: 1/2022

ACEITO EM: 1/2022

PUBLICADO EM: 2/2022 


\section{RESUMEN}

Objetivo: Revisar didácticamente el abordaje general de la Esofagitis Eosinofílica (EEo), discutiendo desde la epidemiología hasta el tratamiento. Revisión bibliográfica: La enfermedad se caracteriza por la acumulación de eosinófilos en el esófago y tiene una mayor incidencia en adultos. La fisiopatología de la EoE está ligada a factores genéticos, ambientales y alergénicos, que atraen eosinófilos y linfocitos Ta la región. Las células T auxiliares 2 se estimulan para producir interleucinas, que activan los eosinófilos y estimulan la producción de eotaxina-3 y reducen la producción de filagrina y desmogleína 1, lo que debilita la barrera epitelial y facilita la inflamación. Los niños experimentan dificultades de crecimiento y aumento de peso, mientras que los adultos experimentan disfagia, regurgitación y plenitud. El diagnóstico se realiza por la presencia de 15 eosinófilos en al menos una de las seis muestras de biopsias esofágicas realizadas por vía endoscópica. La primera línea de tratamiento son los inhibidores de la bomba de protones y los corticosteroides junto con las dietas. La dilatación mecánica por endoscopia y el uso de biológicos son necesarios cuando el paciente no responde a la terapia. Consideraciones finales: La EoE es una enfermedad de difícil diagnóstico con la presencia de síntomas asociados a la Enfermedad por Reflujo Gastroesofágico. Cuando está presente, debe tratarse rápidamente como una complicación.

Palabras clave: Esofagitis eosinofílica, Gastroenterología, Diagnóstico.

\section{INTRODUÇÃO}

A Esofagite Eosinofilia (EEo) é uma doença caracterizada pelo acúmulo de eosinófilos no epitélio esofágico. Seu diagnóstico, realizado por métodos clínicos, histológicos e laboratoriais, necessita de uma investigação de causas primárias a eosinofilia, como a Doença do Refluxo Gastroesofágico (DRGE). Devido a sua cronicidade, o monitoramento contínuo é o principal fator protetor de suas complicações, evitando assim, estenoses esofagianas, as quais permitem com que haja uma impactação do alimento no órgão, dificultando a deglutição e, mais ainda, a qualidade de vida do indivíduo (VIEIRA GG, et al., 2019).

Sua epidemiologia é bastante discutida, uma vez que a disseminação e o acesso aos melhores métodos diagnósticos possibilitaram o atendimento populacional. Dessa forma, ao comparar a dados antigos, verificase uma variação significativa nos últimos anos, com uma elevação de sua incidência, principalmente em adultos, chegando até mesmo, em menor número, nas crianças. Todavia, tal aumento também pode se caracterizar não só pelo número dos diagnósticos realizados, mas também pelo maior acometimento da população (CANARIAS AG, 2018).

Por apresentar um caráter recidivante, descobrir sua etiologia e possíveis fatores que desencadeiam sua sintomatologia são a base para o início do tratamento. Apesar de apresentar sintomas similares a DRGE, na EEo as manifestações clínicas variam de acordo com a idade do indivíduo. Lactentes e pré-escolares apresentam principalmente desenvolvimento ponderal prejudicado, além de vômitos, dor abdominal e recusa de alimentos sólidos. Enquanto nos escolares e adolescentes, as principais queixas são relacionadas à disfagia, com impacto alimentar, disfagia e dor torácica (CANARIAS AG, 2018; PIEDADE S e GASPAR A, 2009; SOCIEDADE BRASILEIRA DE PEDIATRIA (SBP), 2018; SOUZA DFS, et al., 2019).

O tratamento desta enfermidade está relacionado a uma melhora da qualidade de vida provocada pela remissão da doença e, consequentemente, da sintomatologia do paciente. Inibidores da bomba de prótons e corticosteroides tópicos e/ou sistêmicos são usados como primeira linha de tratamento, além de dietas classificadas como elementares, de eliminação e de restrição. As dilatações esofágicas realizadas por via endoscópica são realizadas em não responsivos ao tratamento farmacológico e dietético e que permanecem sintomáticos com estenose do órgão (CANARIAS AG, 2018; NUNES BA, 2018; SOCIEDADE BRASILEIRA DE PEDIATRIA (SBP), 2018).

Este artigo teve como principal objetivo uma revisão sobre a abordagem da EEo, contribuindo para a literatura médica, englobando a epidemiologia, fisiopatologia, os exames complementares necessários tanto para o diagnóstico quanto para o acompanhamento e, porfim, o tratamento desta enfermidade. Visando uma melhor atualização e aprofundamento no assunto tanto para médicos, quanto para estudantes ainda em processo de formação. Espera-se que este artigo seja uma forma de expressar que a EEo é uma doença 
importante, com aumento da sua prevalência e com caráter bastante recidivante, e que, quanto maior aprendizado sobre tal assunto, melhor será a condução do médico com pacientes com diagnóstico positivo.

\section{REVISÃO BIBLIOGRÁFICA}

Esta revisão narrativa foi dividida em 6 blocos, sendo eles: Epidemiologia, Fisiopatologia, Manifestações clínicas, Exames complementares, Diagnóstico e Tratamento, visando um melhor entendimento e organização do pensamento sobre o assunto em questão.

\section{Epidemiologia}

A EEo é uma enfermidade que vem sendo reconhecida nas últimas 2 décadas. Estudos com diversas abordagens metodológicas evidenciam o aumento progressivo de sua incidência e de sua prevalência. Entretanto, não há evidencias claras se esse aumento citado é resultado do real aumento da incidência ou devido ao maior conhecimento e aumento do número de diagnósticos da doença (MENDONÇA LP, 2020; PINTO FM, 2020; SOUZA DFS, et al., 2019; FERREIRA CT, et al., 2019).

Estimativas mostram uma incidência de 7,7 casos para cada 100.000 habitantes por ano em adultos e 6,6 casos para cada 100.000 habitantes por ano em crianças, com uma incidência mundial variando de 5 a 10 casos por 1.000 .000 de pessoas. A doença acomete principalmente homens em uma proporção de 3 a 4:1 para mulheres, com predomínio em países desenvolvidos e em indivíduos caucasianos (RIBEIRO LBM, 2020; SOCIEDADE BRASILEIRA DE PEDIATRIA (SBP), 2018).

Dados mostram prevalência maior em adultos do que em crianças $(34,4$ casos para cada 100.000 habitantes por ano, sendo 42,2 em adultos e 34,4 em crianças.), tendo a faixa etária pediátrica acometida principalmente entre 5 e 10 anos de idade. (RIBEIRO LBM, 2020; SOCIEDADE BRASILEIRA DE PEDIATRIA (SBP), 2018; SOUZA DFS, et al., 2019).

\section{Fisiopatologia}

A EEo ainda guarda muitos mistérios quanto a sua fisiopatologia. Alguns mecanismos da resposta imune durante o processo de inflamação e curso da doença foram explicados, entretanto ainda há dúvidas quanto aos mecanismos que ef etivamente deflagram o início de doença. Fatores genéticos, ambientais e, até mesmo, alguns fatores alérgenos podem estar envolvidos no processo de ativação da imunidade, com atração de células do sistema inato e do sistema adaptativo, entre elas os eosinófilos e linfócitos Te, assim, deflagrar a doença propriamente dita (BALLART MJ, et al., 2020; BENTO ML, et al. 2020; SOUZA DFS, et al., 2019).

Sabe-se que o principal mecanismo imunológ ico envolvido na EEo é mediada por células Thelper 2 (Th2). Os fatores externos estimulam as células Th2 na produção de interleucinas (IL) como as IL-4, IL-5 e IL-13. Essas ILs promovem quimiotaxia e ativação para eosinófilos, mastócitos, basófilos e células iNKT. As principais interleucinas citadas no mecanismo da fisiopatologia são as IL-5 e IL-13. Essas moléculas atuam estimulando a produção de eotaxina-3 e reduzindo a produção de moléculas como a filagrina e desmogleína 1. Esse mecanismo ativado favorece o enfraquecimento da barreia de epitélio do esôfago e facilita o processo inflamatório e a ativação das outras células, como principalmente os mastócitos (BALLART MJ, et al., 2020; BENTO ML, et al. 2020; SOUZA DFS, et al., 2019).

A eotaxina-3 acaba por ser a molécula mais relacionada com a EEo e estudos tem mostrado sua relação com a concentração de eosinófilos encontrados em biopsias esofágicas. Tendo em vista os mastócitos, que são capazes de promover a inflamação e fibrose esofagiana pela liberação de TGF- $\beta 1$ e histaminas, a longo prazo, são capazes de provocarem grandes repercussões no curso da doença (SOCIEDADE BRASILEIRA DE PEDIATRIA (SBP), 2018; MACEDO KV, et al., 2021)

\section{Manifestações clínicas}

A apresentação clínica da EEo varia de acordo com a faixa etária do doente, mas costuma-se manifestar através de sintomas que se assemelham a DRGE, como disfagia, regurgitação alimentar, dor abdominal e plenitude gástrica (XAVIER GA, et al., 2021). 
Geralmente, lactentes e pré-escolares apresentam um quadro clínico mais semelhante com o DRGE, apresentando vômitos, dor abdominal, engasgos e, predominantemente, recusa ou dificuldade de introdução de alimentos sólidos e má progressão estatoponderal. Nos escolares é comum vômitos, epigastralgia, regurgitação alimentar e pirose. No adolescente e no adulto, o impacto alimentar, disfagia e dor to rácica são as queixas habituais (CANARIAS AG, 2018; PIEDADE S e GASPAR A, 2009; SOCIEDADE BRASILEIRA DE PEDIATRIA (SBP), 2018; SOUZA DFS, et al., 2019).

Além das manifestações esofágicas, estudos recentes apontam que a EEo pode apresentar sintomas extra-gastrointestinais como tosse, disfonia e pigarro, reportando acometimento fatingolaríngeo. Queixas otológicas como otalgia sem febre, sensação de plenitude auricular e zumbido também estão presentes, bem como a sintomatologia nasal de obstrução/congestão nasal, prurido nasal, rinorreia posterior e dor facial (BENTO ML, et al., 2020).

\section{Exames complementares}

A Endoscopia Digestiva Alta (EDA) com biópsia é um exame complementar essencial para estabelecer o diagnóstico de Esofagite Eosinofílica, além de monitorar a progressão e remissão da doença, é capaz de verificar a eficácia do tratamento, documentar e dilatar as estenoses e avaliar a recorrência dos sintomas. A doença não possui achados patognomônicos na EDA e, muitas das alterações encontradas, também são associadas a DRGE (CANARIAS AG, 2018).

Os achados endoscópicos incluem sulcos ou estrias verticais, anéis concêntricos fixos ou transitórios, friabilidade da mucosa, eritema e perda da vascularização normal do esôfago, exsudatos esbranquiçados ou placas brancas, mucosa em "papel crepom", lacerações e estreitamento ou estenose da luz esofágica. Todavia, os anéis concêntricos, linhas verticais e placas brancas sãos sinais que apresentam maior sugestividade para EEo (CANARIAS AG, 2018; DIAS EM, et al., 2012).

Importante ainda ressaltar que a associação entre a presença de achados endoscópicos e a confirmação histológica da doença é baixa, fazendo com que ainda haja controvérsias do quanto o endoscopista pode basear seus achados a fidedignidade diagnóstica, logo, somente os achados endoscópicos não são suficientes para diagnóstico. Como resultado, a biópsia mesmo em áreas esofágicas sem alteração, se torna imprescindível para confirmação de diagnóstico (CANARIAS AG, 2018; BARBOSA JPC, et al., 2019).

De ponto de vista histológico a mucosa esofágica é a única do trato gastrointestinal onde não se encontra eosinófilos em condições não patológicas. Na EEo, o esôfago apresentará inflamação eosinofílica em sua mucosa (SOCIEDADE BRASILEIRA DE PEDIATRIA (SBP), 2018).

Não obstante, a contagem dos eosinófilos pode ser subestimada devida sua localização superficial, por isso a preparação da lâmina deve ser bem orientada. Com coloração de rotina com Hematoxilina e Eosina, a infiltração eosinofílica é facilmente notada e frequentemente na doença, os eosinófilos podem ser observados formando microabcessos. (NUNES BAP, 2018; PIEDADE S e GASPAR A, 2009; DIAS EM et al., 2012). Ademais, nas biópsias podem ser encontrados o aumento dos espaços intracelulares no epitélio pavimentoso estratificado, hiperplasia da zona basal, hipertrofia da camada muscular e fibrose da lâmina própria (NUNES BAP, 2018; RIBEIRO LBM, 2020).

\section{Diagnóstico}

Confirma-se a EEo por meio de EDA com 4 a 6 biópsias dos 3 terços esofagianos, sendo eles, os terços superior, médio e inferior, realizando geralmente 2 biópsias por cada região, mesmo não tendo nenhum tipo de alteração visual. A quantidade está indicada devido a forma irregular de infiltração da doença no órgão acometido. É indicada ainda, a realização conjunta de biópsias do estômago e duodeno para a confirmação de eosinófilos presentes apenas no esôfago, pelo fato de não haver eosinófilos presentes na mucosa saudável (CANARIAS AG, 2018; JÚNIOR TCR, 2019; SOUZA DFS, et al., 2019; PEREIRA AD, et. al., 2019).

Além da presença de 15 eosinófilos por campo de grande ampliação em pelo menos uma amostra, para se concluir os critérios do First International Gastrointestinal Eosinophil Research Symposium (FIGERS) e confirmar o diagnóstico, espera-se manifestações clínicas da enfermidade, exclusão dos diagnósticos 
diferenciais, sendo o principal a DRGE, e, por fim, a exclusão de presença de eosinófilos em outras partes do trato gastrointestinal como estômago e duodeno (CANARIAS AG, 2018).

\section{Tratamento}

O tratamento da EEo baseia-se na mitigação da inflamação esofágica, de modo a atenuar a sintomatologia, evitar possíveis complicações, como a condução do tecido esofágico à remodelação e à fibrose, e, consequentemente, proporcionar maior qualidade de vida ao paciente. Essa terapêutica pode ser mediada por drogas, isto é, por Inibidores da Bomba de Prótons (IBP) e por corticosteroides, por dietas, por dilatação mecânica e por medicamentos biológicos (NUNES BA, 2018).

Atualmente, os IBP são a primeira opção terapêutica para os pacientes acometidos pela Esofagite Eosinofilica, sendo utilizados, de maneira geral, em doses de 20 a $40 \mathrm{mg}$ em adultos e de $1 \mathrm{mg} / \mathrm{kg} / \mathrm{dose}$ em crianças de $12 \mathrm{em} 12$ horas em um período de 8 a 12 semanas. Os IBP atuam no bloqueio do fator STAT6, interrompendo a via JAK-STAT mediada pela IL-13 e, consequentemente, reprimindo a produção de eotaxina3 , o que culmina em um ef eito anti eosinofílico. Ademais, agem restaurando a integridade da mucosa esofágica, dificultando, assim, o transporte e entrada de proteínas antigénicas (CANARIAS AG, 2018; NUNES BA, 2018; SOCIEDADE BRASILEIRA DE PEDIATRIA (SBP), 2018).

Apesar de hoje compor a primeira linha terapêutica no tratamento da EEo, a resposta aos IBP era considerada, nas diretrizes publicadas em 2007, fator de exclusão diagnóstica. Isso porque a melhoria no quadro clínico em virtude da administração terapêutica desses medicamentos era considerada sugestiva de doença do refluxo gastroesofágico. Contudo, estudos apontam que grande parte dos pacientes com EEo são responsivos ao tratamento com tal classe de drogas, não havendo distinções clínicas, endoscópicas e histológicas entre os pacientes responsivos e os não responsivos (CANARIAS AG, 2018; NUNES BA, 2018).

A partir da descrição de casos responsivos ao tratamento com inibidores da bomba de prótons, foi criada uma nova classe diagnóstica: a Esofagite Eosinofílica responsiva ao IBP (EEo PPI-R). Porém, devido à incapacidade de distinguir pacientes com EEo e com EEo PPI-R, novos estudos e consensos científicos sugerem que a denominação "EEo PPI-R" é inadequada, pois baseia-se somente na resposta ao tratamento com um fármaco, não considerando, portanto, a responsividade ao IBP como critério de exclusão diagnóstica da EEo (CANARIAS AG, 2018; NUNES BA, 2018; SOCIEDADE BRASILEIRA DE PEDIATRIA (SBP), 2018).

Outras opções terapêuticas eficazes no tratamento da EEo e que também compõem a primeira linha de medicamentos indicados, são corticosteroides sistêmicos e tópicos que proporcionam melhora clínicohistológica, sendo os corticosteroides sistêmicos utilizados em situações emergenciais devido ao risco de potenciais ef eitos colaterais em virtude da necessidade de repetição do uso decorrente da reincidência da doença após a suspensão farmacológica (SOCIEDADE BRASILEIRA DE PEDIATRIA (SBP), 2018; XAVIER GA, et al., 2021).

O tratamento dietético mostra-se bastante eficaz em pacientes com EEo, especialmente em crianças, e fundamenta-se na causa da patologia: a resposta inflamatória imunomediada, existindo três opções nessa modalidade de tratamento: a dieta elementar, as dietas de eliminação e as dietas de restrição. A dieta elementar mostrou-se eficaz na maioria dos pacientes, culminando na remissão sintomatológica e na melhora histológica, mas o custo, a palatabilidade e a recorrência sintomática após a reintro dução de algumas proteínas alimentares são potenciais empecilhos para adoção dessa opção terapêutica. Nas dietas de eliminação, opta-se por eliminar das refeições alimentos ligados a repostas alérgicas, como leite, ovo, peixes, mariscos, frutos secos, amendoim, soja e trigo. Outra opção nesse sentido é a restrição alimentar com base em avaliação alergológica, a qual é realizada mediante testes de hipersensibilidade (CANARIAS AG, 2018; SOCIEDADE BRASILEIRA DE PEDIATRIA (SBP), 2018; MACEDO KV, et al., 2021).

O procedimento endoscópico com dilatação é indicado para pacientes que não obtiveram resposta à terapêutica farmacológica ou dietética e que apresentam sintomatologia intensa, com estreitamento esofágico e estenose que causem impactação alimentar, havendo melhoria sintomática temporária por não atenuar a inflamação esofágica, sendo inevitável reintervenção (CANARIAS AG, 2018; NUNES BA, 2018; SOCIEDADE BRASILEIRA DE PEDIATRIA (SBP), 2018). 
Estudos abordam tratamentos experimentais com anticorpos monoclonais anti-TNF- $\alpha$, anti-IL-5 e anti-lgE, em virtude de suas ações potencializadoras da patologia, não obtendo, porém, benefícios clínicos significativos (NUNES BA, 2018; SOCIEDADE BRASILEIRA DE PEDIATRIA (SBP), 2018).

A resposta ao tratamento deve ser verificada não somente mediante a melhora sintomatológica, mas também por meio da realização de endoscopias e biópsias de controle, de modo a verificar alterações clínicas, endoscópicas e histológicas a partir da instituição e/ou alteração de uma opção terapêutica (CANARIAS AG, 2018).

\section{CONSIDERAÇÕES FINAIS}

A EEo é uma doença que está evidenciando-se em constante aumento, principalmente entre adultos. Sua fisiopatologia ainda é muito controversa e há incerteza no que se diz respeito a abertura do quadro, podendo ter como gatilho fatores genéticos, ambientas e até mesmo fatores alérgenos. Quanto as manifestações clínicas, estas podem variar de acordo com o intervalo de idade do doente, sendo comumente associadas as mesmas formas expressas na DRGE. Simultaneamente, sintomas extra esofágicos, otológicos e nasais podem ser encontrados. Na investigação da patologia, achados endoscópicos são ineficientes para confirmação e a biópsia com análise histopatológica torna-se padrão ouro. Com a validação da doença, o tratamento é feito com uso de IBP e corticoides tópicos ou sistêmicos, além de contar com trat amento dietético em conjunto. O presente estudo faz-se de grande valia para educar sobre essa patologia que acomete diferentes faixas etárias e é capaz de acarretar prejuízos funcionais e na qualidade de vida dos pacientes, principalmente quando atrelado ao diagnóstico tardio.

\section{REFERÊNCIAS}

1. BALLART MJ, et al. Esofagitis eosinofílica: diagnóstico y manejo. Rev Med Chile, 2020;148, 831-841.

2. BARBOSA JPC, et al. Association between endoscopic findings and histopathological confirmation in patients with suspicion of eosinophilic esophagitis, Arq Gastroenterol, 2019;56(2): 151-154

3. BENTO ML, et al. Manifestações ORL em crianças com Esofagite Eosinofílica. Revista Portuguesa De Otorrinolaringologia E Cirurgia De Cabeça E Pescoço, 2020; 58(4):205-211.

4. CANARIAS AG. Esofagite Eosinofilica. Dissetação (mestrado integrado em medicina) - Clínica Universitária de Otorrinolaringologia. Universidade de Lisboa, Lisboa, 2018, 10-13.

5. DIAS EM, et al. Esofagite eosonofílica: Atualização e contribuição da endoscopia. Boletim Científico de Pediatria, $2012 ; 1(1): 22-25$.

6. FERREIRA CT, et al. Eosinophilic esophagitis --- Where are we today?. Jornal de Pediatria, 2019; 95(3): 275-281.

7. JÚNIOR TCR. Esofagite eosinofílica e o uso de corticoides tópicos: revisão sistemática. Dissertação (Graduação em Medicina). Centro Universitário UNIFACIG, Manhuaçu, 2019;29.

8. MACEDO KV, et al. Uso da dieta de eliminação no tratamento de Esofagite Eosinofílica: uma revisão de literatura. Research, Society and Development, 2021;10(13):e588101321552.

9. MENDONÇA LP, Pinto FM. Esofagite eosinofílica na pediatria:um conceito em evolução. Brazilian Journal of Health Review, 2020;3(1):375-381.

10. NUNES BAP, Esofagite Eosinofílica - Uma Revisão Fisiopatológica. Dissertação (mestrado integrado em medicina) - Faculdade de medicina. Universidade de Coimbra. Coimbra, 2018.

11. PEREIRA AD, et. al. Esofagite eosinofílica: entidade clínica emergente ou subdiagnosticada?.Revista Caderno de Medicina,2019;2(1):206-211

12. PIEDADE S e GASPAR A. Esofagite eosinofílica. Revista portuguesa de imunologia, 2009;17(3):215-224

13. RIBEIRO LBM. Achados Endoscópicos Sugestivos De Esofagite Eosinofílica Correspondem à Eos inofilia No Esôfago Em Pacientes Pediátricos?, PR. Dissertação (Mestrado em Medicina interna) - Setor de Ciências da Saúde. Universidade Federal do Paraná, Curitiba, 2020;51 p.

14. SOCIEDADE BRASILEIRA DE PEDIATRIA (SBP). Departamento Científico de Gastroente rologia, Guia Prático de Atualização. Esofagite Eosinofílica. 2018. Disponível em: https://www.sbp.com.br/fileadmin/user_upload/20035gGPA - Esofagite_Eosinofilica_final-marco.pdf. Acessado em: 8 de jan. de 2022.

15. SOUZA $\bar{A}$ DSS, et al. Esofagite eosinofílica em pacientes pediátricos: uma revisão de literatura. Revista Eletrônica Acervo Saúde, 2019;29: e1092.

16. VIEIRA GG, et al. Endoscopic and histological characteristics in patients with eosinophilic esophagitis responsive and non-responsive to proton pump inhibitors. Jornal de Pediatria, 2019. 95(5): 638-643

17. XAVIER GA, et al. Farmacoterapia no tratamento da esofagite eosinofílica: comparando anticorpos monoclonais e corticoesteróides. Uma revisão sistemática. Brazilian Journal of Development, 2021;7(7):67088-67111. 